\title{
Ecoulement instationnaire dans un diffuseur aubé d'une pompe centrifuge
}

\author{
Unsteady flow field in a vaned centrifugal pump diffuser
}

par K. Eisele, F. Muggli, Z. Zhang, M.V. Casey

Sulzer Innotec

This paper describes experimental and computational work to examine the unsteady flow field in the vaned diffuser of a medium specific speed pump. The aim of this project is to verify a commercialy available Navier-Stokes program with a $k$ - $\varepsilon$ turbulence model and to improve our understanding of the complex unsteady three-dimensional flow field associated with the interaction between a pump impeller and its vaned diffuser.

The paper provides the results of experiments carried out using Laser Doppler Anemometry (LDA), in which timeresolved details of the unsteady flow field in a vaned diffuser of a medium specific speed pump have been obtained as a function of the local position of the pump impeller blades. The static pressures were also measured at the hub and shroud side of the diffuser.

The measurement results have been analysed to elucidate some interesting flow features observed in this typical pump diffuser. These include unsteady recirculation of the flow from the diffuser into the impeller at partload and flow separation in the diffuser channel. The unsteady flow simulation of a two dimensional diffuser showed the capabilities and the limits of a Navier Stokes program with $k-\varepsilon$ turbulence model. The comparisons of the simulation results with experimental data identify that this simple computational model with a time periodic inlet profile is able to show important flow phenomena. In terms of the flow field and pressure rise there is no substantial difference between the predictions of a steady simulation and the time-average of an unsteady simulation.

\section{INTRODUCTION}

Les méthodes numériques pour le calcul de l'écoulement sont de plus en plus importantes pour le développement et le dimensionnement des turbomachines [1]. La simulation de l'écoulement dans la roue à l'aide de logiciels Navier-Stokes est devenue dès le début un outil efficace pour le développement et le dimensionnement des roues de pompes [2]. Ces simulations ont pu être fortement améliorées grâce à une plus grande capacité des ordinateurs mais aussi par des connaissances plus approfondies, par exemple en tenant compte des pertes par fuite [3]. C'est ainsi que par le moyen du logiciel Navier-Stokes il a été possible de déterminer à l'avance, de façon exacte, l'instabilité pour une roue à nombre de tours spécifique élevé [4].

Les expériences positives faites avec le logiciel NavierStokes pour le calcul d'un écoulement tridimensionnel et stationnaire dans la roue, ont conduit à essayer la simulation de l'écoulement dans le diffuseur, ce qui représente un deuxième pas vers la simulation de l'étage entier. Étant donné que l'aubage fixe du diffuseur est placé après la roue mobile, l'écoulement dans le diffuseur est donc fortement influencé par l'écoulement à la sortie de la roue. Par consé- quent l'écoulement est fortement tridimensionnel et instationnaire, ce qui rend très difficile la définition des conditions aux limites. D'autre part le calcul d'un écoulement décéléré à l'aide du modèle de turbulence "k $\varepsilon^{\text {", }}$, dont les points faibles sont connus, est également difficile [5], spécialement parce que la meilleure récupération de pression dans le diffuseur a lieu dans le voisinage de la zone où débute le décollement instationnaire.

Pour décrire de façon satisfaisante cet écoulement complexe avec une simulation numérique, il faut procéder progressivement. D'une part il est nécessaire d'examiner le diffuseur dans une pompe sur le banc d'essais avec des mesures détaillées de la pression et de la vitesse. Ces essais fourniront une compréhension approfondie de l'écoulement dans le diffuseur, mais aussi les données nécessaires pour l'optimisation de la simulation. D'autre part, en ce qui concerne la simulation numérique, il faut aussi procéder progressivement. En premier lieu, la simulation numérique a été mise au point pour un écoulement stationnaire dans un diffuseur tridimensionnel. Puis l'interaction instationnaire entre les aubes de la roue mobile et celles du diffuseur a été examinée avec une simulation bidimensionnelle du diffuseur.

Les chapitres suivants donnent d'abord la description du 
banc d'essais et des systèmes de mesure. Ensuite sont décrites les diverses simulations du diffuseur. Une comparaison des résultats entre les mesures et les simulations et leur évaluation, est présentée à la fin de cet article.

\section{II — BANC D'ESSAIS ET SYSTÈME DE MESURE}

Ces analyses ont dû être effectuées sur un banc d'essais spécialement conçu pour des mesures optiques (Fig.1). Cette installation a permis la mesure détaillée des pressions statiques et instationnaires le long des parois du diffuseur. 4 points de mesure ont été placés sur la ligne médiane à la paroi côté jante, et 7 à la paroi côté moyeu. Les pressions instationnaires ont été mesurées avec des capteurs piézorésistifs de très faibles dimensions. Les données principales sont reportées dans le tableau No. 1 .

\begin{tabular}{|l|c|}
\hline Diamètre & $2,7 \mathrm{~mm}$ \\
\hline Pression max. & $20 \mathrm{bar}$ \\
\hline Bande de fréquence (3dB) & $100 \mathrm{kHz}$ \\
\hline Sensibilité & $4 \mathrm{mV} / \mathrm{bar}$ \\
\hline Facteur de linéarité & $0,4 \%$ \\
\hline
\end{tabular}

Tableau I. - Données principales des capteurs miniatures.

La vitesse a été mesurée au moyen d'un anémomètre à effet Doppler (ALD) en fonction de la position angulaire du rotor. L'anémomètrie ALD fournit, pour un point choisi, des informations très fines concernant la résolution de la vitesse par rapport au temps [6]. Cette pompe d'essais a été conçue comme un étage intermédiaire d'une pompe multicellulaire. Le diffuseur a été légèrement transformé, par rapport à la géométrie initiale, pour permettre un bon accès optique d'une part, et pour faciliter la définition des conditions aux limites pour la simulation numérique d'autre part. Les données principales de la pompe sont énumérées dans le tableau No. 2. La section du diffuseur s'ouvre aussi dans le sens axial, du côté moyeu ( paroi divergente ). Par ce fait, le diffuseur est fortement chargé. Selon les diagrammes de dimensionnement de Reneau, ce diffuseur se trouve juste dans la zone de transition où a lieu le décollement instationnaire [7].

\begin{tabular}{|l|l|}
\hline Roue mobile & Diffuseur \\
\hline $\mathrm{D} 0=140 \mathrm{~mm}$ & $\mathrm{D} 3=364 \mathrm{~mm}$ \\
\hline $\mathrm{D} 1=208 \mathrm{~mm}$ & $\mathrm{D} 4=496 \mathrm{~mm}$ \\
\hline $\mathrm{D} 2=350 \mathrm{~mm}$ & $\mathrm{D} 5=570 \mathrm{~mm}$ \\
\hline $\mathrm{B} 2=32,9 \mathrm{~mm}$ & $\mathrm{~B} 3=34,5 \mathrm{~mm}$ \\
\hline $\mathrm{n}=1000 \mathrm{U} / \mathrm{min}$ & \\
\hline Nbr. aubes: 7 & Nbr. aubes: 12 \\
\hline
\end{tabular}

Tableau II. - Données principales de la pompe d'essais.

\section{III $\square$ SIMULATIONS DU DIFFUSEUR}

Les simulations du diffuseur sont réalisées à l'aide d'un logiciel Navier-Stokes disponible dans le commerce avec un modèle de turbulence " $k \varepsilon^{\text {". }}$ Ce même programme a aussi été utilisé avec succès pour la simulation de l'écoulement dans des roues de compresseurs, de pompes centrifuges et de turbines.

\subsection{Simulation stationnaire du diffuseur}

L'écoulement à l'entrée du diffuseur est dicté par les conditions d'écoulement à la sortie de la roue mobile. A la sortie de la roue, l'écoulement est fortement tridimensionnel et instationnaire. Les simulations du diffuseur ont été effectuées d'après les équations de Navier-Stokes pour un écoulement tridimensionnel mais stationnaire, car la simulation d'un écoulement instationnaire et tridimensionnel est encore trop coûteuse. La zone considérée par le calcul commence dès la sortie de la roue et s'étend jusqu'au canal qui conduit à l'étage suivant. La figure 2 montre le maillage pour la simulation de la roue mobile et du diffuseur. Étant donné que tous les canaux du diffuseur sont semblables il suffit, pour un calcul stationnaire, de simuler l'écoulement dans un seul canal. La zone qui intéresse le calcul est représentée avec un maillage type " $\mathrm{H}$ " comprenant 68750 nœuds et partagé en plusieurs blocs.

Puisque l'écoulement à l'intérieur du diffuseur dépend essentiellement de l'écoulement à l'entrée du diffuseur, le choix des conditions aux limites à l'entrée du diffuseur est très important.

La moyenne des résultats de la simulation de la roue mobile, prise sur la circonférence de la roue, a été choisie pour déterminer les conditions aux limites. Cela exige une très haute qualité de la simulation de la roue mobile. C'est pour cette raison que parallèlement aux simulations du diffuseur, la simulation de la roue mobile a dû être améliorée par un maillage plus fin et optimalisé. Les améliorations du maillage ont permis une meilleure définition des arêtes d'entrée et de sortie des aubes de la roue mobile, ce qui a conduit à de bien meilleurs résultats.

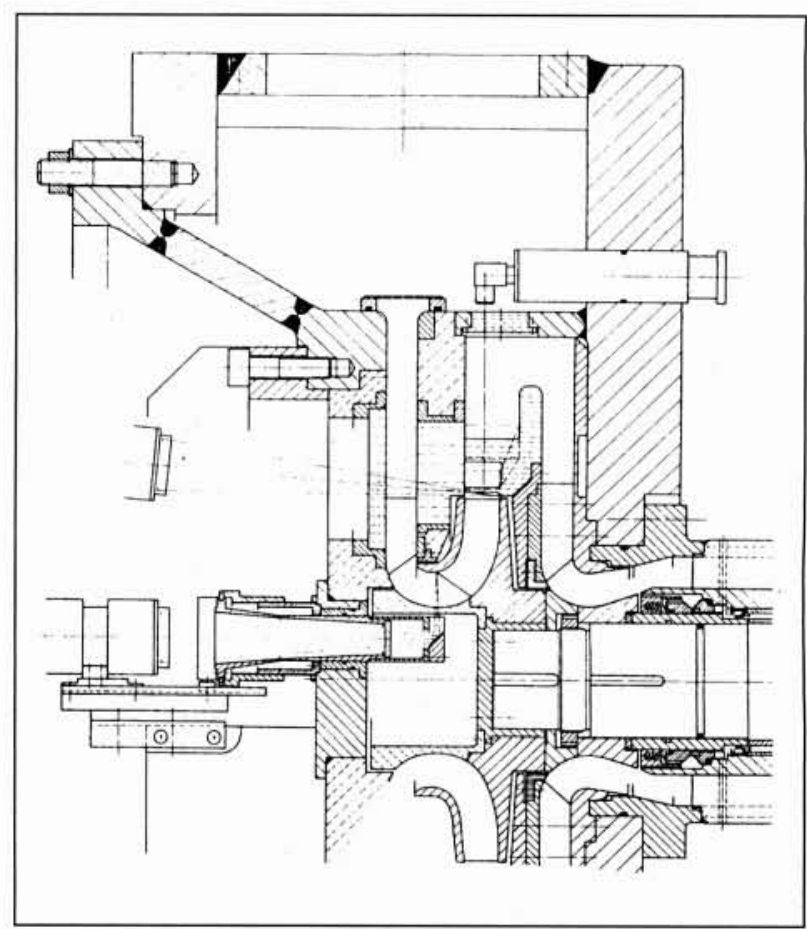

1. Pompe d'essai avec diffuseur aubé. 


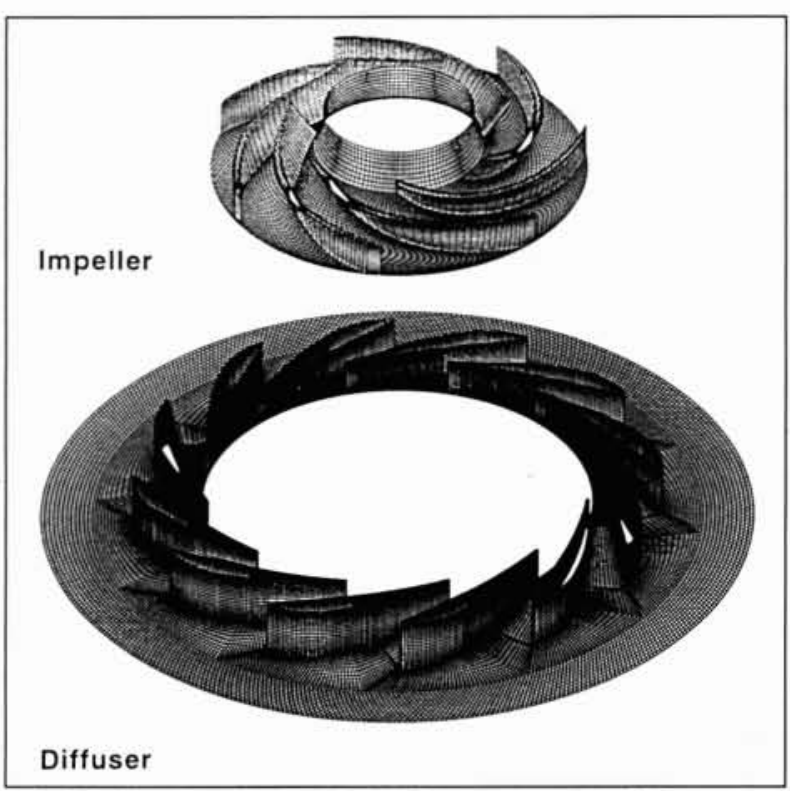

2. Maillage pour le calcul d'une pompe radiale : roue mobile et diffuseur.

\subsection{Simulation instationnaire du diffuseur}

Pour la simulation instationnaire de l'écoulement dans le diffuseur, on a utilisé le même maillage que pour la simulation stationnaire. Puisqu'il s'agit d'abord d'une simulation quasi-bidimensionnelle, le maillage a été limité à 3 coupes radiales. Par contre 2 canaux du diffuseur ont été pris en considération. Les conditions aux limites, à l'entrée du diffuseur, sont déterminées par les pressions totales et les angles de la vitesse, en fonction de l'angle de rotation du rotor. Ces valeurs ont été calculées à partir des pressions instationnaires mesurées à la paroi et des vitesses détectées au moyen de

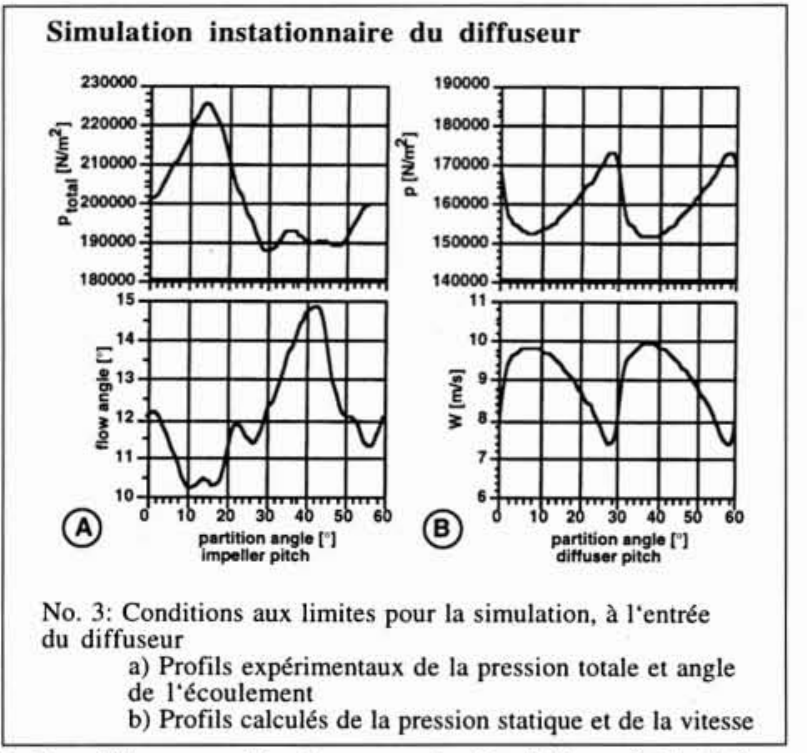

3. Conditions aux limites pour la simulation, à l'entrée du diffuseur.

a) Profils expérimentaux de la pression totale et angle de l'écoulement

b) Profils calculés de la pression statique et de la vitesse.

l'anémomètre ALD. L'analyse de Fourier a livré des fonctions qui ont servi à décrire la variation des valeurs en fonction du temps ou de l'angle de rotation. Ces fonctions de la pression totale et de l'angle de la vitesse, décrivent le passage d'un canal de la roue mobile. Il faut remarquer que pour avoir des conditions de périodicité favorables, le nombre effectif des aubes mobiles a été diminué de 7 à 6 . Les pressions totales et les angles des vitesse sont représentés dans la figure $3 \mathrm{a}$. La variation des pressions statiques et des vitesses à l'entrée du diffuseur, dans la figure $3 \mathrm{~b}$, sont le résultat de la simulation. L'influence des aubes à l'entrée du diffuseur est très marquée aux points d'impact à $30^{\circ}$ et à $60^{\circ}$.
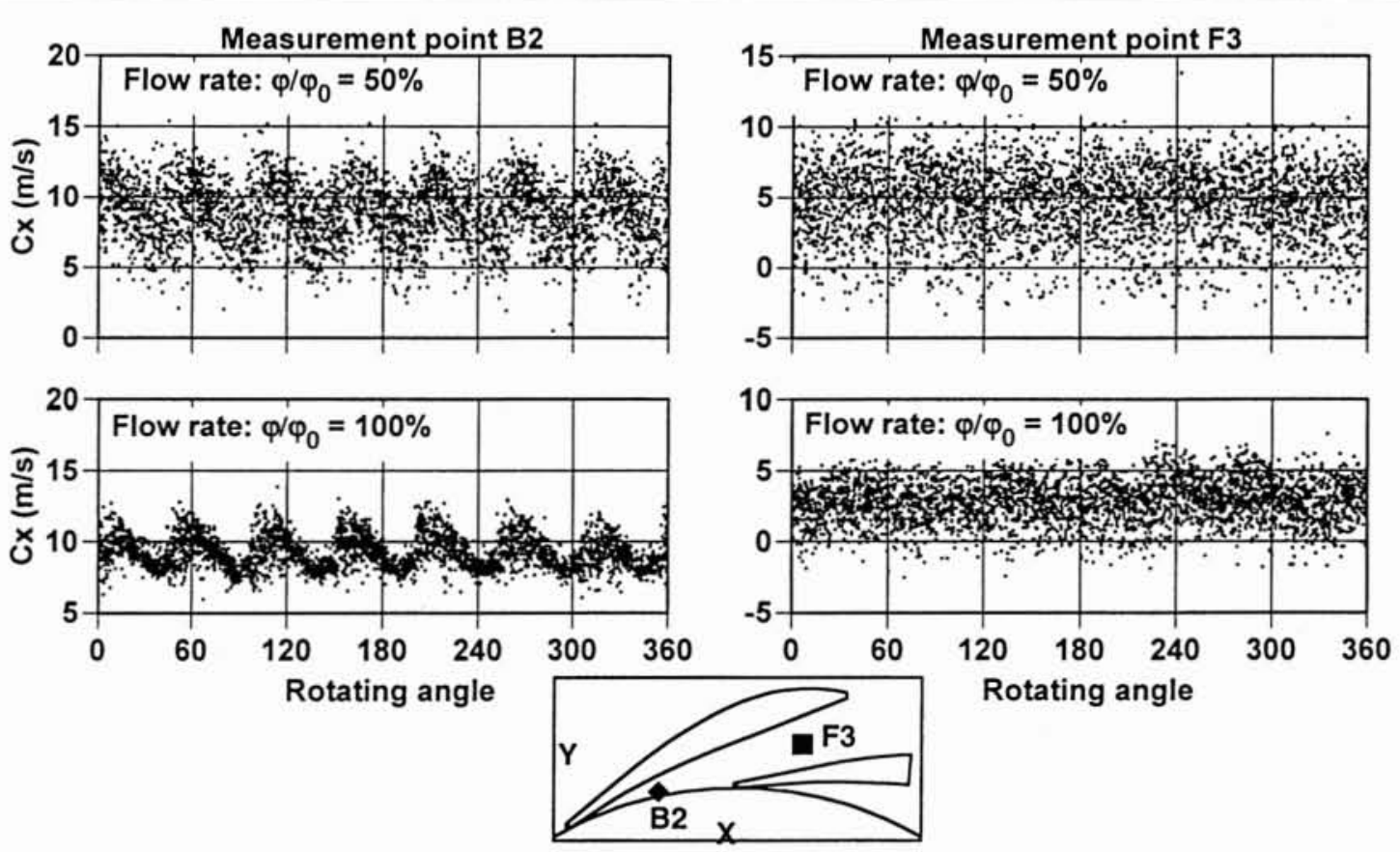

4."Scatter diagrams" des vitesses mesurées à l'aide de ALD en 2 points de mesure et pour 2 débits. 


\section{IV — RÉSULTATS DE L'ANALYSE EXPÉRIMENTALE}

Une première représentation de quelques mesures de vitesse montre la complexité de l'écoulement.

Les mesures à l'aide de l'anémomètre à effet Doppler (ALD) ont permis de déterminer le vecteur vitesse, dans ses trois dimensions, en fonction du temps. Chaque mesure ALD est composée d'environ 20000 à 30000 mesures individuelles qui peuvent être attribuées à un certain angle de rotation de la roue mobile. Cela permet d'ordonner les mesures de la vitesse en fonction de l'angle de rotation. La figure 4 montre environ 5000 mesures individuelles de la composante " $\mathrm{X}$ " en fonction de l'angle de rotation, en deux points du diffuseur et pour deux débits différents. La composante indiquée présente, à peu de chose près, la même direction que l'écoulement principal. Ces nuages de points ne sont pas dus à l'inexactitude de la mesure, mais à la turbulence périodique de l'écoulement. Les fluctuations périodiques, engendrées par l'aubage de la roue mobile, sont très marquées à l'entrée du diffuseur — point de mesure B2dans les deux points de fonctionnement. Ces fluctuations périodiques diminuent le long du canal du diffuseur. Par contre les fluctuations dues à la turbulence, traduites par la grande dispersion des points, augmentent fortement le long du canal du diffuseur (Point de mesure F3). Les fluctuations périodiques, dues à l'aubage de la roue mobile, deviennent presque invisibles à charge partielle, car le caractère de l'écoulement est alors dicté par d'autres phénomènes instationnaires.

Les valeurs moyennes de la vitesse et des fluctuations sont calculées à partir des valeurs reportées sur la figure 4 . On peut former des moyennes partielles ou globales, pour une position angulaire donnée. Les valeurs des 3 composantes de l'écoulement reportées sur la figure 5, sont des moyennes partielles calculées pour une ouverture d'angle de rotation de $3^{\circ}$. Les fluctuations périodiques des 3 composantes de la vitesse, clairement visibles sur la figure, suivent la même fréquence que l'aubage mobile. Les valeurs moyennes partielles de la variation de vitesse (Rms) sont pratiquement les mêmes pour les points de mesure B2 et F3, de la figure 5 . Les valeurs Rms de la troisième composante n'ont pas été déterminées pour des raisons techniques.

La vitesse radiale à la sortie de la roue, particulièrement en ce qui concerne la formation de contre-courant à charge partielle, joue un rôle prépondérant pour la stabilité de la caractéristique de la pompe. Pour cette raison elle a été mesurée et analysée en plusieurs points de fonctionnement. La figure 6 montre les vecteurs de la vitesse, mesurés au point $\mathrm{C} 1$, en fonction de la largeur de la roue pour tous les points de fonctionnement analysés. Chaque ligne de la figure 6 représente les résultats d'un point de fonctionnement. Les colonnes, par contre, donnent les résultats pour différentes positions de la roue mobile. Ces positions sont indiquées schématiquement au-dessus des colonnes. La 5ème colonne donne la moyenne globale pour le point de mesure $\mathrm{Cl}$.

$\mathrm{Au}$ point de meilleur rendement de la pompe $(\mathrm{Q} / \mathrm{Qo}=100 \%)$ il ne se forme jamais de contre-courant dans le point de mesure $\mathrm{C} 1$. A charge partielle $\mathrm{Q} / \mathrm{Qo}=68 \%$, il y a un début de contre-courant côté jante, mais pas encore pour toutes les positions de la roue mobile. Pour le point de fonctionnement $\mathrm{Q} / \mathrm{Qo}=50 \%$, la zone de recirculation passe du côté du moyeu. Le contre-courant est maintenant établi pour toutes les positions de la roue mobile. La valeur moyenne globale, dans la région du moyeu, est aussi négative et le vecteur est dirigé contre la roue. Pour des charges partielles en dessous de $\mathrm{Q} / \mathrm{Qo}=50 \%$, il y a des zones de contre-courant aussi bien du côté de la jante comme du côté du moyeu. Le passage de la zone de contre-courant d'un côté à l'autre de la roue a une grande importance en ce qui concerne la stabilité de la caractéristique de la pompe.

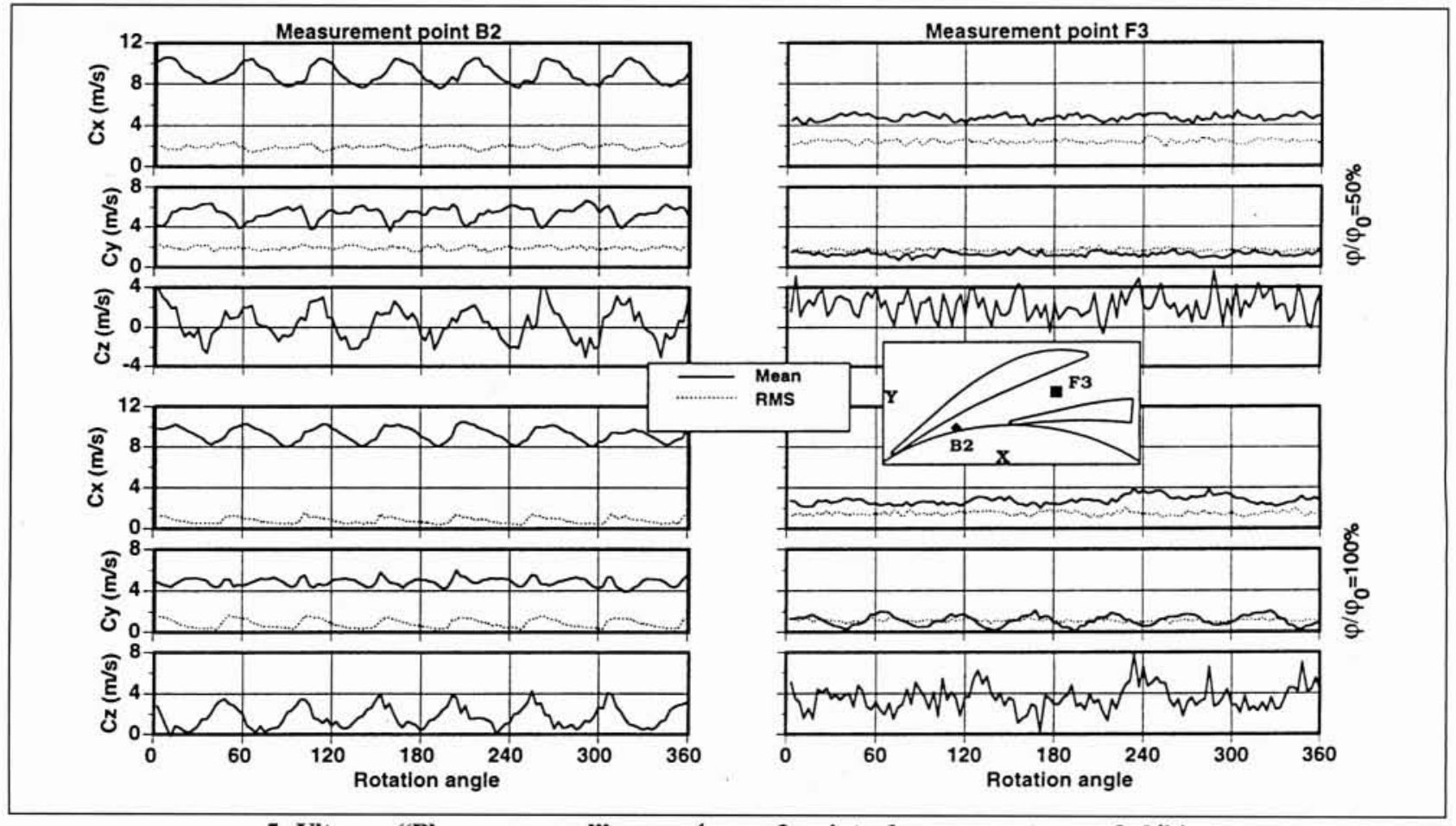

5. Vitesses "Phase-averaged" mesurées en 2 points de mesure et pour 2 débits. 


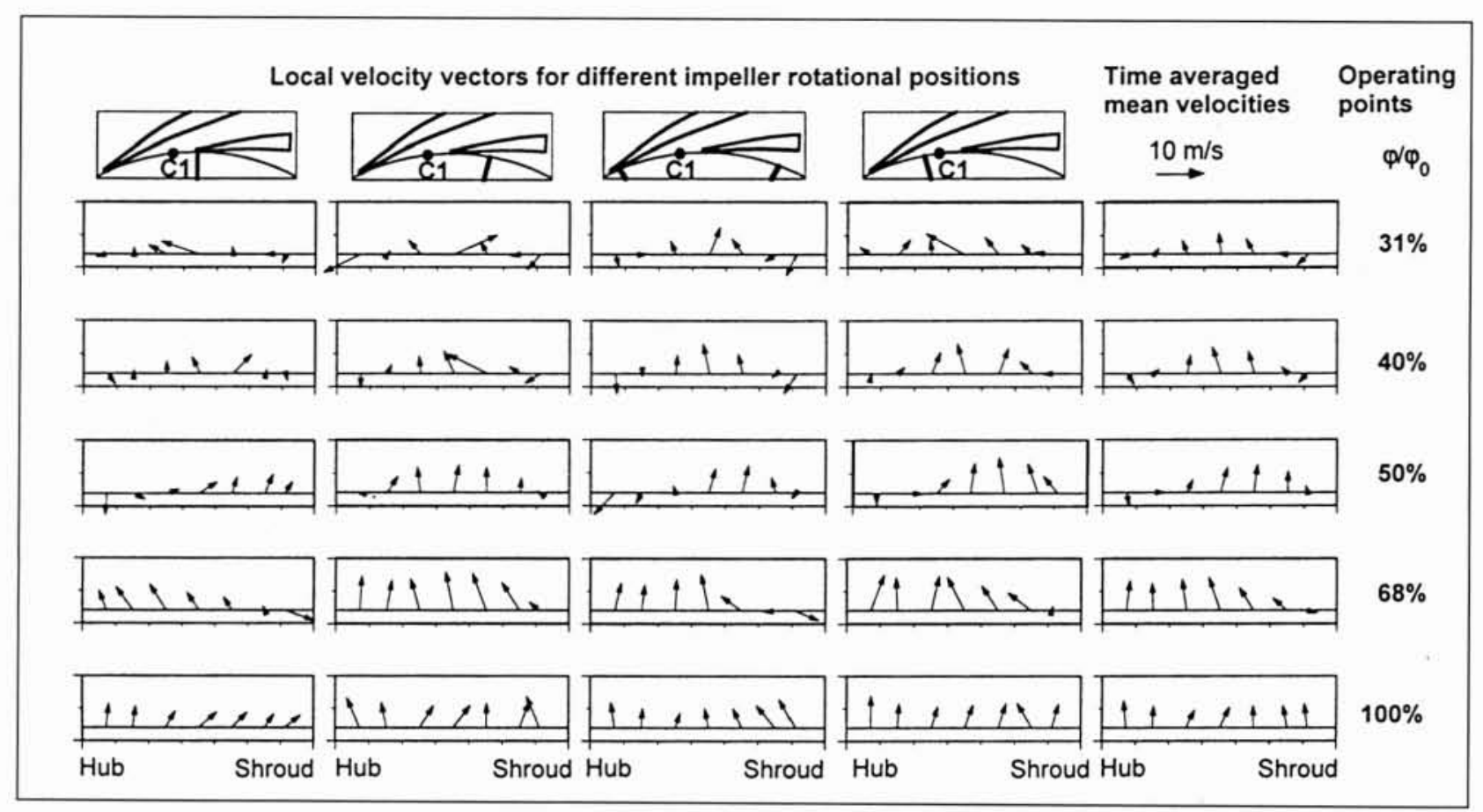

6. Vecteurs de la vitesse méridienne, à la sortie de la roue mobile et pour des points de fonctionnement différents.

La grandeur de la zone de contre-courant dépend aussi de l'endroit de la mesure par rapport au pas du diffuseur. La plus grande zone se trouve à proximité de l'arête de l'aube du diffuseur et à son intrados. Les valeurs moyennes globales acquises au moyen de l'anémomètre ALD seront prises en considération pour la comparaison avec les résultats de la simulation numérique et seront discutées dans le chapitre suivant.

\section{V — RÉSULTATS DES SIMULATIONS}

\section{- 5.1 Résultats des simulations stationnaires et compa- raison avec les mesures}

Les vitesses calculées dans le diffuseur peuvent être comparées directement avec les valeurs moyennes globales mesurées à l'aide du ALD. Dans la figure 7 les vecteurs de la vitesse sont représentés dans une vue en plan sur les aubes du diffuseur à 3 niveaux différents. La différence de structure de l'écoulement d'un niveau à l'autre, montre bien que l'écoulement est fortement tridimensionnel. A l'endroit où la paroi du diffuseur, côté moyeu, devient divergente, il y a décollement du courant. Dans les 3 simulations numériques, il y a décollement du courant à proximité de la paroi côté moyeu. Ce décollement, qui n'est pas visible dans les vecteurs ALD, existe en réalité. Cela a été démontré par la visualisation de l'écoulement dans la pompe [6]. Le décollement est en effet instationnaire en ce qui concerne sa position et son apparition. Les très grandes variations de la vitesse mesurées à l'aide de ALD (Rms $>0,4^{*}$ mean) sont un indice d'amorçage de décollement à la paroi côté moyeu. Le début du décollement peut être clairement déterminé dans une section moyenne du canal du diffuseur (Fig. 8). Les mesures ALD s'accordent bien avec les simulations numériques. Des résultats ALD n'existent pas pour la zone de décollement proprement dite.

La qualité des simulations peut aussi être contrôlée à l'aide de la récupération de pression dans le diffuseur. La figure 9 montre l'augmentation de pression, rendue adimensionnelle selon l'équation ci-dessous, le long de la ligne médiane du diffuseur.

$$
\psi=\frac{p_{\text {lokal }}-p_{\operatorname{Re} f}}{\rho / 2 * u_{2}^{2}}
$$

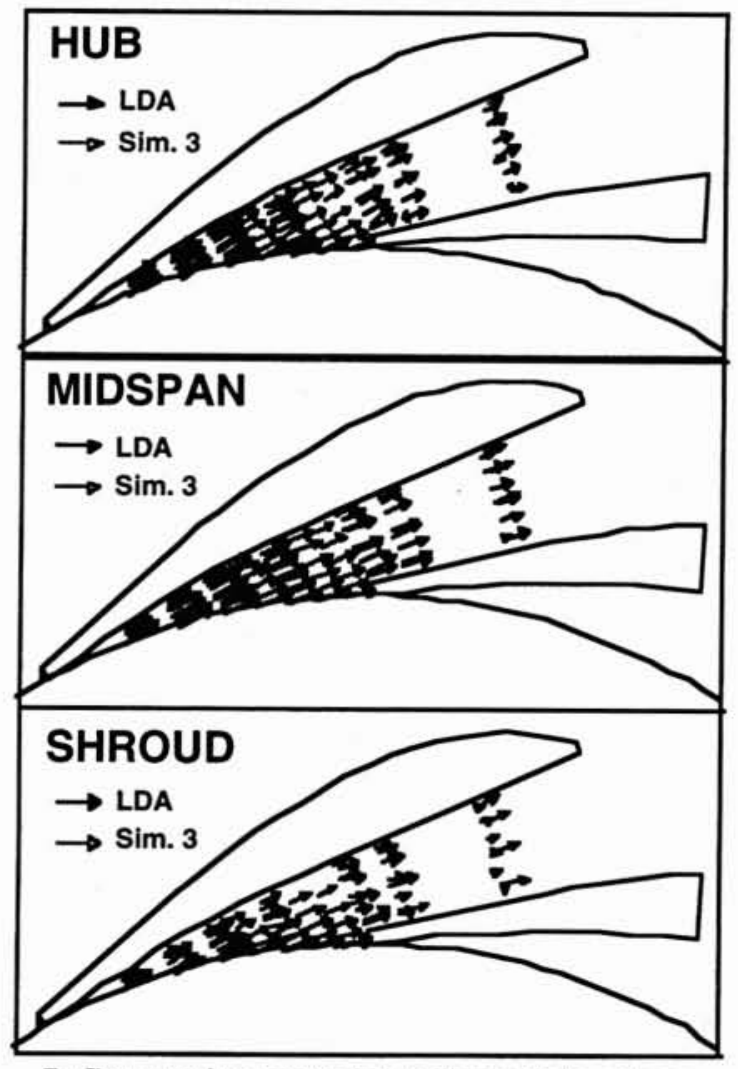

7. Comparaison entre les vecteurs de la vitesse mesurés (ALD) et les calculés. 


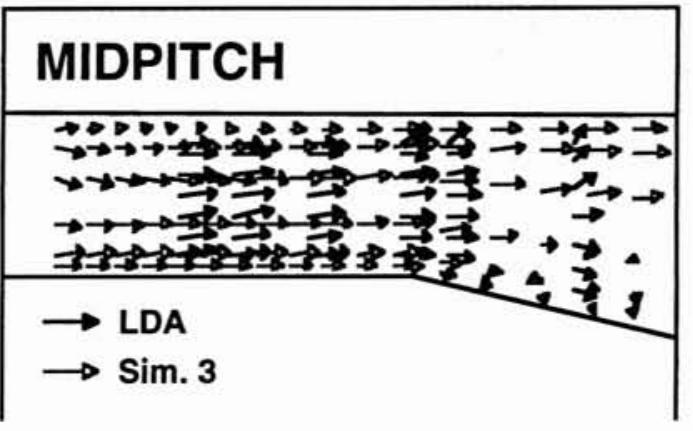

8. Comparaison entre les vitesses mesurées et calculées, dans la ligne médiane.

La pression mesurée à l'entrée du diffuseur, au milieu du canal et à la paroi côté jante, a été prise comme pression de référence. Jusqu'à la cote $S=0,082$ la concordance avec les mesures est assez bonne. C'est à cet endroit que prend naissance le décollement ; dès lors, pour les simulations, la récupération de pression est très faible. La zone de recirculation correspond, en ce qui concerne la simulation, à une zone d'obstruction. Ainsi en dehors de cette zone il se forme des vitesses élevées, d'où peu d'augmentation de pression. Comme il a été dit plus haut, le décollement est instationnaire, d'où la différence entre la pression mesurée et celle obtenue des simulations.

\subsection{Résultats des simulations instationnaires et compa- raison avec les mesures}

Les valeurs moyennes, par rapport au temps, des résultats de la simulation instationnaire sont d'abord comparés avec les résultats de la simulation stationnaire au milieu du canal (Fig. 10). Cette comparaison montre qu'il n'y a aucune différence pour la zone comprise entre l'entrée du diffuseur et la section la plus étroite. En aval de ce point il se forme de petites différences, qui grandissent dans la région qui suit les aubes du diffuseur. La plus grande différence se trouve dans le sillage des aubes. Dans le cas de la simulation stationnaire, cette différence est plus faible.
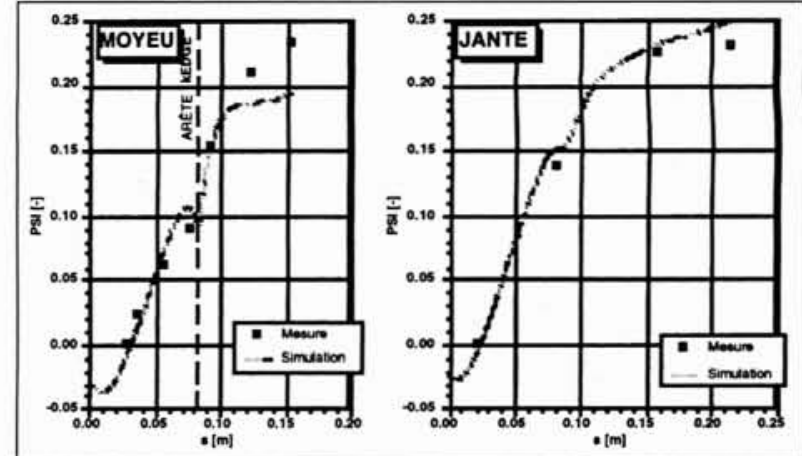

9. Augmentation de la pression statique, le long de la ligne médiane du diffuseur.

La prédiction de la structure de l'écoulement et de la récupération de pression est pratiquement la même pour les cas stationnaire et instationnaire. La simulation stationnaire peut donc être employée pour le dimensionnement des diffuseurs de turbomachines radiales. La simulation d'un étage complet avec "mixing plane" est suffisante pour la détermination de l'écoulement stationnaire dans la machine.

En comparant les résultats de la simulation instationnaire avec ceux des mesures, il faut tenir compte du fait que la simulation est bidimensionnelle tandis que les mesures sont tridimensionnelles. Cela conduit naturellement à un certain écart des résultats. Afin de limiter cet écart, seules les mesures faites au milieu du canal ont été prises en considération pour la comparaison.

La comparaison entre les vecteurs vitesse calculés et ceux mesurés, pour 4 différentes positions de la roue mobile, est donnée dans la figure 11. Jusqu'à la section la plus faible, les résultats correspondent très bien. Particulièrement la direction du courant, donc l'angle d'entrée dans le diffuseur, peut être calculée correctement. En aval de la section la plus faible, les résultats commencent à s'écarter. Cela est dû au fait que le caractère de l'écoulement est fortement tridimensionnel dans cette zone. C'est dans cette même zone que les résultats expérimentaux ont montré un décollement de l'écoulement.

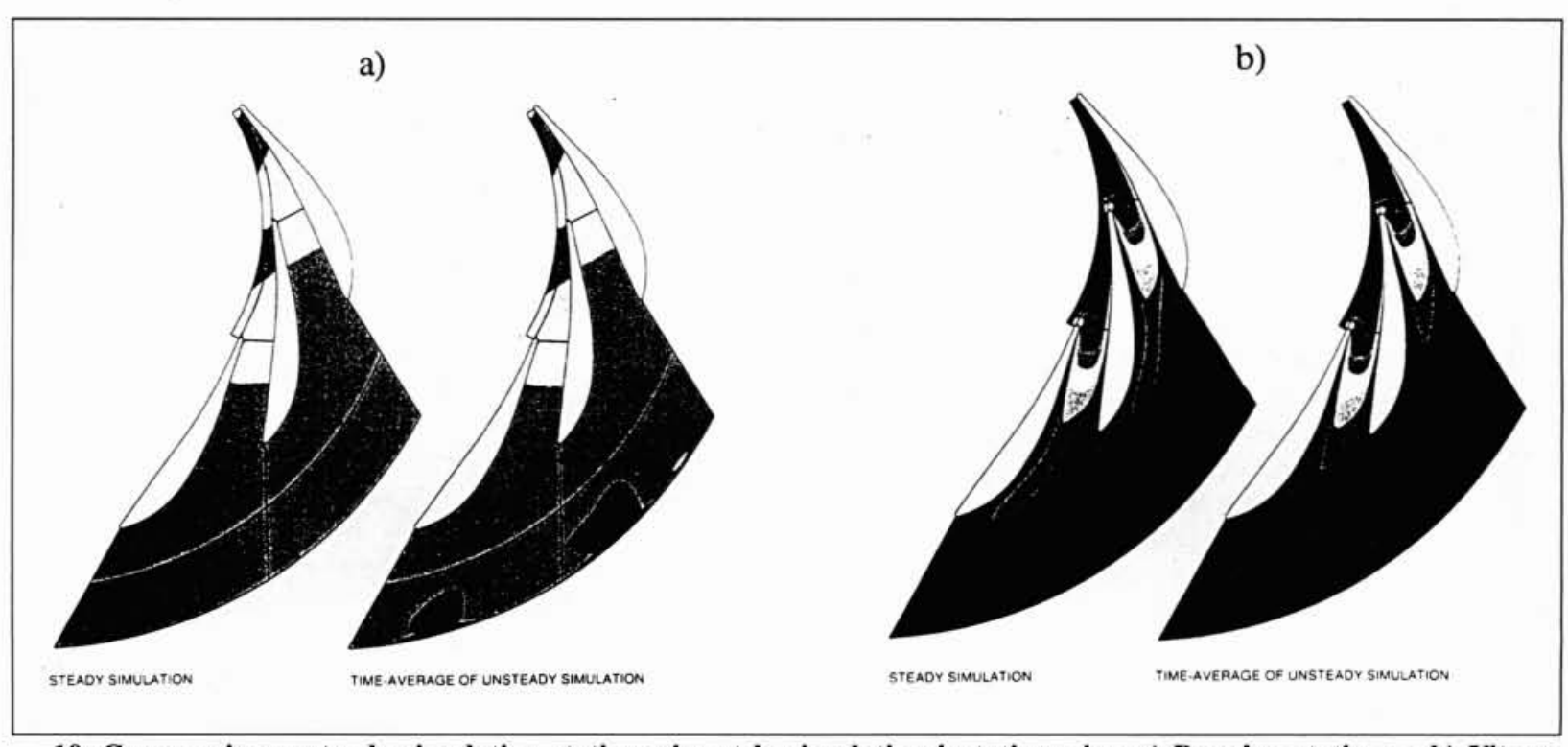

10. Comparaison entre la simulation stationnaire et la simulation instationnaire. a) Pression statique ; b) Vitesse. 


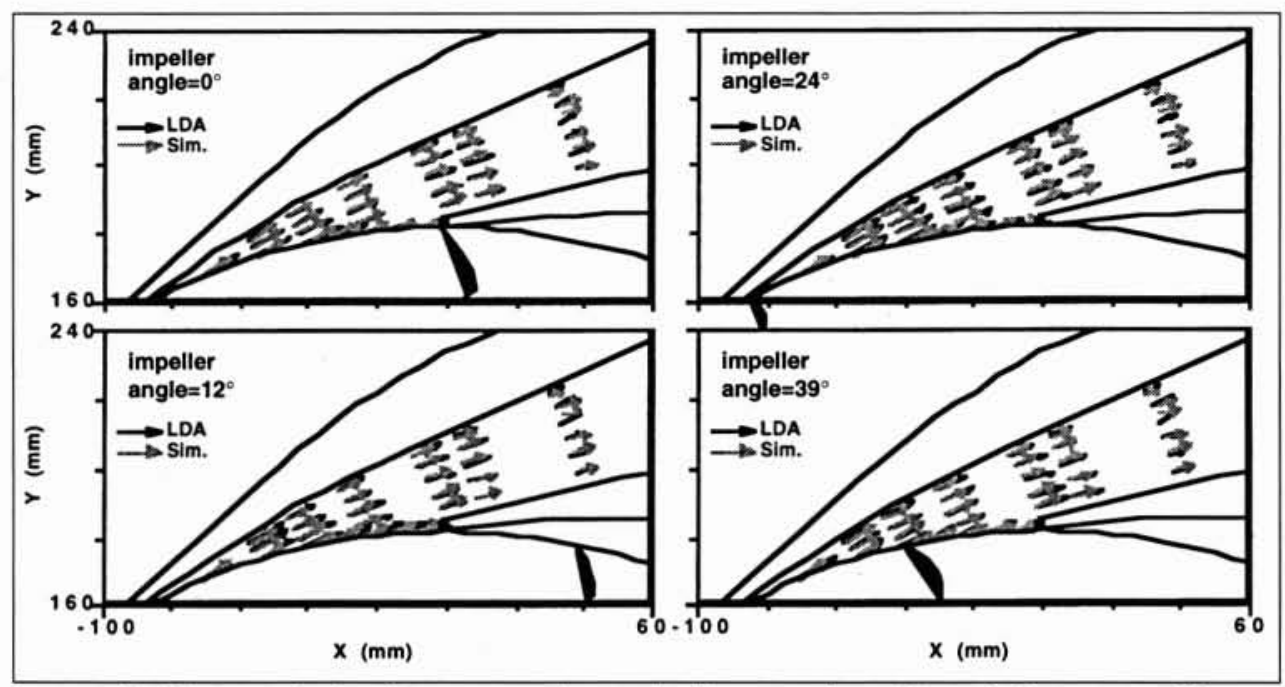

11. Vecteurs de la vitesse mesurée et calculée pour 4 positions de la roue mobile. l'amplitude de ses variations, diminuent le long du diffuseur. Dans la plupart des cas, les vitesses mesurées sont plus élevées que les valeurs calculées.

Comme la figure 13 le montre, la simulation des pressions statiques correspond mieux aux résultats de mesure des pressions instationnaires. Aux points de mesure 1,2 et 4 , sur la ligne médiane du diffuseur, les pressions à la paroi ont été mesurées du côté jante comme du côté moyeu. Les variations de la pression sui-

L'anémomètre ALD a permis de mesurer les vitesses, et leur variation, en 6 points de mesure, le long de la ligne médiane du diffuseur et au milieu du canal. Ces valeurs sont comparées, dans la figure 12 , avec les résultats de la simulation pour différentes positions de la roue mobile. Bien que l'analogie des résultats dans cette région ne soit pas très bonne, les mesures et le calcul montrent que la vitesse et vent clairement la fréquence qui correspond au passage des aubes de la roue mobile, le long du diffuseur.

$$
\Delta p^{*}=\frac{\Delta p}{\rho / 2 * u_{2}^{2}}
$$

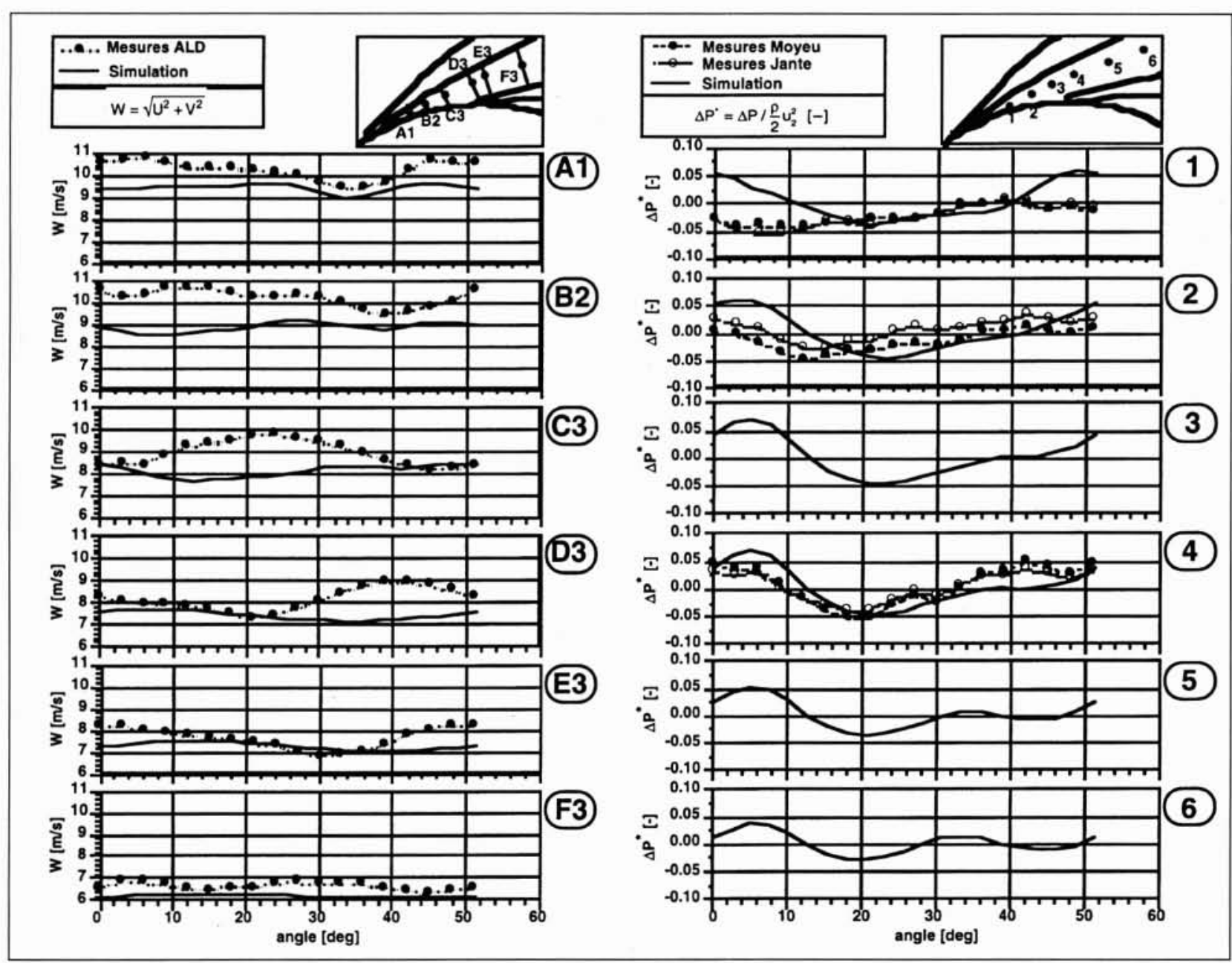

12. Vitesses mesurées et calculées le long de la ligne médiane du diffuseur.
13. Pressions mesurées et calculées le long de la ligne médiane du diffuseur. 
Mais leur amplitude diminue en direction de la sortie.

Les résultats de la simulation instationnaire bidimensionnelle correspondent qualitativement assez bien avec les mesures. Les deux montrent que le sillage des aubes de la roue mobile, tout en diminuant d'intensité, traverse tout le diffuseur.

\section{VI $\square$ CONCLUSION}

Les mesures tridimensionnelles à l'aide de l'anémomètre ALD ont montré que l'écoulement dans le diffuseur est fortement tridimensionnel et instationnaire. Des essais de visualisation de l'écoulement ont montré qu'il se forme un décollement instationnaire à l'intrados des aubes du diffuseur du côté du moyeu.

Dans les simulations stationnaires du diffuseur on retrouve le décollement du côté du moyeu. Par contre la zone de décollement produite par la simulation est sensiblement plus grande que la zone mesurée. Etant donné que l'augmentation de pression dans le diffuseur dépend de l'ampleur de la zone de décollement, la correspondance entre les pressions mesurées et celles obtenues des simulations est bonne avant tout dans la région où l'écoulement n'est pas perturbé.

Malgré que le modèle de turbulence "k $\varepsilon$ " ne permette pas une prédiction exacte du point où prend naissance le décollement, la simulation numérique est un outil très utile pour le développement des diffuseurs.

Les moyennes, par rapport au temps, des résultats de la simulation de l'écoulement instationnaire dans le diffuseur aubé, ont été comparés avec les résultats des simulations stationnaires. On a constaté de bonnes correspondances entre les deux cas. Pour le dimensionnement des pompes radiales, il suffit donc d'effectuer des simulations stationnaires pour l'étage roue-diffuseur. Pour cela l'écoulement stationnaire de chaque composante est calculé séparément, pour être ensuite relié à l'aide de "mixing plane".

Cette analyse a montré que les simulations stationnaires sont suffisamment bonnes pour le dimensionnement, au niveau industriel, des diffuseurs de pompes. Par contre, pour des études plus poussées (p. ex. "rotating stall"), il faut alors avoir recours aux calculs instationnaires qui sont plus coûteux.

\section{Remerciements}

Les auteurs remercient les entreprises Sulzer Pompes SA, Sulzer Hydro, Sulzer Turbo et Sulzer Innotec qui ont contribué financièrement à cette étude et qui ont permis la publication de cet article. Ils remercient également Mr. A. Crua pour la traduction.

\section{BIBLIOGRAPHIE}

[1]. Casey M.V.: The industrial use of CFD in the design of Turbomachinery, AGARD Lecture Series Turbomachinery design using CFD, AGARD LS-95, 1994.

[2]. Schachenmann A., J.F. Gülich : Vergleich von drei Navier-Stokes Berechnungsverfahren mit LDA-Messungen an einem radialen Pumpenlaufrad. Fachbeitrag Pumpentagung Karlsruhe 6.bis 8. Okt. 1992

[3]. Eisele K., F. Muggli, D. Wiss, Z. Zhang : Improving Pump Development with Laser and Computer. Chem. Technology Europe, November/December 1994.

[4]. Holbein P., K. Kaupert, Th. Staubli : Computational Fluid Dynamics in a pump of high specific speed. ASC-User Conference Germany, Aying 1995.

[5]. Wilcox, D.C.: Comparison of two-equation turbulence models for boundary layers with pressure gradient. AIAA Journal, Vol. 31. No8,pp1414-1421.

[6]. Eisele K., Z. Zhang, F. Muggli : Investigation of the unsteady diffuser flow in a radial pump. 7 th Int. Symp. on Appl. of Laser Techniques to Fluid Dynamics, 11.7.-14.7.1994, Lisbon, Portugal.

[7]. Reneau L.R., J.P. Johnston, S.J. Kline : Performance and Design of Straight Two Dimensional Diffusers, ASME J. of Basic Engineering. March 1967, PP 141-150.

[8]. Muggli F. A., D. Wiss, K. Eisele, Z. Zhengji, M. V. Casey : Unsteady flow in the vaned diffuser of a medium specific speed pump, Proc. of the 1996 Int. Gas Turbine and Aeroengine Congress \& Exhibition, 10-13.June 1996, ASME-Paper 96-GT-157, New York. 